\title{
Grounding Interventionism: Conceptual and Epistemological Challenges Amanda Bryant
}

\section{This is a draft of a paper forthcoming in Metaphilosophy. Please cite the published version when available.}

\begin{abstract}
Philosophers have recently highlighted substantial affinities between causation and grounding, which has inclined some to import the conceptual and formal resources of causal interventionism into the metaphysics of grounding. The prospect of grounding interventionism raises two important questions: exactly what are grounding interventions, and why should we think they enable knowledge of grounding? This paper will approach these questions by examining how causal interventionists have addressed (or might address) analogous questions and then comparing the available options for grounding interventionism. I argue that grounding interventions must be understood in worldly terms, as adding something to or deleting something from the roster of entities, or making some fact obtain or fail to obtain. I consider three bases for counterfactual assessment: imagination, structural equation models, and background theory. I conclude that grounding interventionism requires firmer epistemological foundations, without which the interventionist's epistemology of grounding is incomplete and ineffectually rationalist.
\end{abstract}

Keywords: grounding interventionism, causal interventionism, epistemology of metaphysics, metametaphysics, imagination, intuition, counterfactual reasoning, modal epistemology

\section{Introduction}

Philosophers have recently drawn substantive connections between grounding and causation, by drawing attention to their shared formal, metaphysical, and epistemic features. ${ }^{1}$ Grounding is standardly taken to be an asymmetric metaphysical dependence relation that either is explanatory or backs explanation, wherein some derivative fact or entity obtains or exists in virtue of some more fundamental fact or entity. ${ }^{2}$ Grounds are also standardly (though not universally) taken to metaphysically necessitate that which they ground. ${ }^{3}$ Typical examples include: Socrates grounds Singleton Socrates, the fact that $\mathrm{P}$ and the fact that $\mathrm{Q}$ together ground the fact that $\mathrm{P} \wedge \mathrm{Q}$, the fact that $\mathrm{P}$ grounds the fact that PvQ, God's love grounds the pious (or vice versa), natural features

\footnotetext{
${ }^{1}$ See Schaffer (2016) and A. Wilson (2018a, 2018b, forthcoming). For critical responses, see Bernstein (2016) and Koslicki (2016). See also Wang (2020) for discussion.

${ }^{2}$ For introductions to grounding, see Audi (2012), Fine (2012), Raven (2015), Rosen (2010), Schaffer (2009). On the connection between grounding and explanation, see Dasgupta (2017), Glazier (2020), Maurin (2015), and Thompson (2016).

3 See deRosset (2010), Rosen (2010), and Trogdon (2013). For dissenting views, see Leuenberger (2014) and Skiles (2015).
} 
ground moral features, truthmakers ground truths, and fundamental physical facts ground biological facts.

Grounding is normally thought to differ from causation in some key respects. For instance, grounding can relate abstract relata, is synchronic, and relates more fundamental relata to less fundamental ones. By contrast, causation only relates concrete physical events, is diachronic, and typically relates equally fundamental relata (inter-level causation being an unusual and contested sort of case). Nevertheless, philosophers have also emphasized a number of features in common. Grounding and causation are both standardly thought to be irreflexive, asymmetric, transitive metaphysical dependence relations of a productive nature, intimately bound up with explanation and aptly captured by counterfactual conditionals.

Those who emphasize the parallels between causation and grounding have attempted to extend the conceptual and formal resources of interventionism to grounding. For instance, A. Wilson claims:

The deep structural similarity between grounding and causation suggests that the asymmetry of grounding is reflected in an asymmetry of consequences of interventions. If we intervene to change the ground fact, the grounded fact changes. If we intervene to change the grounded fact, the ground fact is unchanged. (A. Wilson forthcoming, 18)

According to this line of thought, interventionist conditionals capture important features of grounding. Similarly, Schaffer uses interventionist language to describe a test for grounding claims: "there is a straightforward and informative... working test of token grounding to be had, in terms of counterfactual covariation: wiggle the ground, and the grounded wiggles" (Schaffer 2016, 74). So there is some initial inclination in the literature to enfold grounding under the interventionist rubric. Call the resulting view (to be precisified below) grounding interventionism.

Causal interventionists face foundational challenges to the articulation and defence of their view, which have been well explored in metaphysics and philosophy of science. ${ }^{4}$ They include, most prominently, the challenge of cashing out the central notion of an intervention in a way that is not viciously circular. Nevertheless, causal interventionism remains an attractive theoretical alternative in certain respects, partly due to the capacity of counterfactual conditionals to capture and explain a heterogeneous array of cases (Paul 2009), as well as the elegance and power of the formal machinery that accompanies it (Pearl 2000). It is natural to hope that accounting for grounding in interventionist terms might prove similarly fruitful.

However, before getting too optimistic about the prospects for grounding interventionism, a number of substantive questions must be addressed. First, what does it mean to 'wiggle the ground', as it were, and to see if the grounded wiggles? That is, what are grounding interventions, and what do they involve? Second, what are the epistemic credentials of grounding interventionism? That is, is 'wiggling the ground' and seeing what happens a good way to find out about grounding? Can would-be grounding interventionists offer a satisfactory epistemological story about how we can come to know or form justified beliefs about grounding? The aim of this paper is to address both sorts of question.

${ }^{4}$ Hitchcock and Woodward (2003); Meek and Glymour (1994); Pearl (2000); Spirtes, Glymour, and Scheines (2000); Woodward (1997, 2000, 2003); and Woodward and Hitchcock (2003). 
We may begin to do so by looking to antecedent articulations and refinements of the substance of causal interventionism. In particular, to help clarify what it might mean to 'wiggle the ground', we may consider how causal interventionists have characterized 'wigglings'. They standardly characterize the class of causal interventions as not limited to actual manipulations or practically possible ones, since causation is often independent of what we actually do and of what is practically possible for us to do. For example, Woodward points out that past events and large-scale cosmological events are uncontroversially causal, notwithstanding the impossibility of our manipulating them $(2003,10)$. So the relevant class of interventions must include counterfactual interventions. Evaluating the relevant counterfactuals sometimes requires us to countenance far out (that is, modally remote) scenarios. ${ }^{5}$ It will be instructive to attend to this feature of causal interventions, because — as we will see —- grounding interventionism likewise requires us to countenance far out counterfactual scenarios.

Since counterfactuals are so central to causal interventionism, the epistemology of counterfactuals is a natural place to begin considering how the causal interventionist's epistemological story might go. All sorts of philosophical and psychological work has been done on the epistemology of counterfactuals. ${ }^{6}$ I will consider three (mutually compatible) suggestions about what enables and constrains counterfactual reasoning. First, I will consider Williamson's (2007b) epistemology of counterfactuals, which assigns a prominent role to the imagination. On that view, imagination is the key cognitive capacity that allows us to perform the needed counterfactual evaluations, and to wiggle a causal or grounding relatum is an imaginative act. Second, I will consider structural equation models, which formally represent causal and grounding dependencies and potentially illuminate relations among concepts and claims of interest. Finally, I will consider the potential for background theory to constrain counterfactual evaluation. Evaluating the epistemic credentials of grounding interventionism will then require determining whether the epistemic resources at issue - imagination, structural equation models, or theoretical constraints — can adequately constrain our assessments of grounding counterfactuals.

I will argue that when imagination is taken to be the primary basis for evaluating interventionist counterfactuals, interventionist frameworks — whether causal or grounding require us to stretch our imaginative resources beyond their adequacy conditions. In cases where experience doesn't constrain imaginative simulations, imagination calls on antecedent intuitions that lack adequate epistemic footing. If so, then causal interventionism and grounding interventionism alike invoke an epistemically deficient form of modal rationalism. Moreover, I will argue that structural equation models can at best complement an independent epistemology of interventionist counterfactuals, because they require prior knowledge of the structural equations they encode.

\footnotetext{
${ }^{5}$ For convenience, I will occasionally invoke the metaphor of modal distance, but I don't wish to hang much on it, since it suggests a kind of linear progression of difference from actuality (merely logical possibilities always differing most from reality as compared to merely metaphysical possibilities, as compared to merely physical possibilities) that breaks down under scrutiny, with carefully chosen examples. I thank Anand Vaidya for drawing this to my attention.

${ }^{6}$ See, for instance, Byrne (2007), Hoerl et al. (2011), Ichikawa (2011), Kroedel (2012, 2017), Mandel et al. (2005), Schulz (2017), and Williamson (2007a, 2007b).
} 
Lastly, I will argue that holding fixed certain background theories can constrain our evaluation of some interventionist counterfactuals, but that counternomics and countermetaphysicals again force us toward an unacceptably rationalist modal epistemology.

Let me be clear in advance about the strength of my thesis. I will not argue that grounding interventionism renders knowledge of grounding unachievable. I will argue, rather, that the epistemic credentials of grounding interventionism are limited — cognizance of which should temper erstwhile high hopes that the interventionist framework can imbue theories of ground with increased objectivity, greater scientific respectability, or improved capacity for discovery. There are a spectrum of cases running from straightforward and unproblematic cases to intractable ones. My central claim will be that none of the candidate epistemological stories gets us very far past the easy end of the spectrum. Moreover, since grounding theorists don't typically confine themselves to the easy end, the available epistemological options don't go very far toward vindicating metaphysical practice.

Section 2 will describe Woodward's causal interventionism and consider some possible formulations of grounding analogs. Section 3 will detail the causal interventionist's notion of an intervention before using it to guide the articulation of an analogous ground-theoretic notion. Section 4 will concern the epistemology of interventionist counterfactuals. It will address three (potentially complementary) approaches to counterfactual evaluation, respectively involving imagination, structural equation models, and theoretical constraint. At the same time, it will evaluate the capacity of each approach to generate knowledge or justified belief with respect to causation and grounding. Section 5 will conclude.

\section{Interventionisms}

Theories of causation have many different aims - they can concern causation itself, causal patterns, causal explanation, causal discovery and prediction, causal concepts and language (or their use in certain contexts), causal learning and reasoning, effective strategies, and so forth. I will conceive of causal interventionism as a primarily ontological project, i.e. as the project of nonreductively accounting for causation and identifying causal relationships. I will take Woodward's (2003) account as an exemplar. On his account, relationships between variables are causal when (roughly) intervening on one results in a change to the other $(2003,45)$. Assuming from here on that $X$ and $Y$ are not identical:

\section{Causal interventionism:}

$X$ causes $Y$ iff there is a possible intervention on $X$ that changes the value of $Y$.

Woodward qualifies the view in a number of fine-grained ways, which I'll omit in the interests of maintaining a simple and general sense of the view. As I have already noted, since there are salient senses in which interventions need not be possible, the view is also amenable to counterfactual formulation: 

were it carried out, would change the value of $Y$.

A natural place to start when formulating grounding interventionism would be by constructing an exactly parallel view. Such a view would say, roughly, that two variables represent a grounding relation when intervening on one results in a change to the other. Formulated counterfactually, we get the following view. Assuming from here on that $\alpha$ and $\beta$ are not identical:

\section{Grounding interventionism (counterfactual): $\alpha$ grounds $\beta$ iff there is a possible intervention on $\alpha$ that, were it carried out, would change the value of $\beta$.}

Note that I have changed the variables from the causal formulation to reflect potential differences in kind between causal relata and grounding relata. Without any explicit restriction to the ranges of the variables, causal interventionism and grounding interventionism appear to make the precisely the same condition sufficient for causation and for grounding. In fact, they appear to collapse counterfactual dependence with causation and grounding, respectively, in a way that would be immediately implausible to anyone who thinks counterfactual dependence tracks multiple kinds of metaphysical dependence. At any rate, grounding and causation are standardly thought to have incompatible features. In particular, as I mentioned at the outset, grounding is normally thought to be synchronic and causation diachronic; but a relation cannot be both at once, since one relatum is either temporally co-present with another or not. ${ }^{7}$ If that's right, then without further qualification, causal interventionism and grounding interventionism are incompatible, because they entail that any case of causation is a case of grounding and vice versa. This presents a problem for anyone wishing to endorse a package deal of both interventionisms.

However, the ranges of the variables typically are restricted by the aptness conditions of the relevant interventionist models. For instance, Schaffer specifies aptness conditions for causal models and for grounding models, according to which the variables range over events and entities, respectively (2016, $67 \& 74)$. Would-be grounding interventionists who take the relata of grounding relations to be facts would have to formulate that condition differently. If causation and grounding are indeed mutually exclusive, their challenge would be to do so in a way that precludes a single dependency from fitting both the causal and the grounding models. This would presumably require holding fixed a philosophical background theory according to which facts and events are categorially distinct. 8

\footnotetext{
7 I borrow this characterization of the synchronic/diachronic distinction from J. Wilson (in progress), who convincingly argues that the distinction is not between temporal instantaneity and extension but rather temporal co-presence and non-co-presence. It is also worth noting that A. Wilson argues that the apparent distinction between causation and grounding in terms of their respective diachronicity and synchronicity "does not run deep" (2018b, 729).

${ }^{8}$ I thank an anonymous reviewer for encouraging me to consider aptness conditions such as these more explicitly.
} 
An alternative to relying on aptness conditions alone to adequately restrict the application of each interventionist view would be to weaken the views by removing their sufficiency conditions. In the case of grounding interventionism, the resulting view would be as follows:

\section{Grounding interventionism (weak): $\quad \alpha$ grounds $\beta$ only if there is a possible intervention on $\alpha$} that, were it carried out, would change the value of $\beta$.

Since we are not in the business of providing a reductive account, this appears to be a reasonable amendment to the view. However, even this weakened formulation apparently admits of counterexample. For instance, suppose that $\mathrm{P}$ grounds $\mathrm{P} \vee \mathrm{Q}$. It is possible to wiggle $\mathrm{P}$ without the truth of the disjunction wiggling. ${ }^{9}$ Where both $\mathrm{P}$ and $\mathrm{Q}$ are true, $\mathrm{P}$ and $\mathrm{Q}$ each fully grounds the disjunction. This is a case of grounding overdetermination (Koslicki 2015) that is analogous to cases of symmetric causal overdetermination, in which some effect has more than one operative and individually sufficient cause. As such, it is a case of grounding that does not satisfy the necessary condition posited by grounding interventionism (weak). 10

Suppose we stipulate that grounding interventions must wiggle all the putative grounds. That would mean we could not capture as much grounding structure as we would like, since we would not have the means to say that, in cases of symmetric grounding overdetermination, each ground is individually sufficient for grounding. Another response would be to argue that cases of symmetric grounding overdetermination can be bracketed, by suggesting that they are an artifact of a sui generis kind of logical grounding (Correia 2014), which requires an independent account. Yet symmetric grounding overdetermination may not be so easily bracketed. A. Wilson describes it as "commonplace" and gives an example that — to my mind - is not logical: the potion's being poisonous is grounded in its containing 1 gram of arsenic and also in its containing 1 gram of strychnine (A. Wilson 2018b, 743). A further response to grounding overdetermination would be to weaken grounding interventionism still further, so that it posits neither necessary nor sufficient conditions for grounding, but instead says that some counterfactual conditionals track some grounding relations. However, without general principles stating when and why the counterfactual analysis applies, such a view would not be particularly illuminating.

A more promising response might be to selectively negate the influence of any symmetrically overdetermining relata. Woodward suggests that, in cases of symmetric overdetermination, causal interventionists can get the right result for a particular cause $X$ by, roughly, "freezing" the influence of other direct causes and finding that an intervention on $X$ will

\footnotetext{
9 Thanks to Claudio Calosi for raising this counterexample.

10 If the affinities between grounding and causation do indeed run deep, then it isn't surprising that the cases that make trouble for causal interventionism - including symmetric overdetermination and, as we will see below, preemption - make trouble for grounding interventionism, too. Grounding interventionists such as Schaffer and A. Willson acknowledge such problems and hold only that the interventionist framework works for a range of suitably unproblematic cases.
} 
change the value of $Y(2003,83) .{ }^{11}$ This manoeuvre exploits the thought that the causal dependence of $Y$ on $X$ "fails to express itself in counterfactual dependence only because [the other symmetrically overdetermining cause] happens to be present as well" (2003, 83); if we remove the influence of other symmetrically overdetermining causes, we find the appropriate counterfactual dependence. A parallel approach to grounding overdetermination might motivate a revision to grounding interventionism (weak), such that, roughly, the relevant intervention on $\alpha$ would change the value of $\beta$ supposing we have negated the influence of any symmetrically overdetermining grounds.

Even with such a qualification, the initial formulations of grounding interventionism might still be thought to be confused. I have designed them to capture Schaffer's and A. Wilson's thought that grounding interventions target grounds. However, arguably cases of grounding preemption show that this approach sometimes gets the direction of necessity and dependence wrong. I will discuss grounding preemption in greater detail in section 4.2. The gist of the problem is that grounded facts or entities can have multiple full grounds that perform their grounding role only when the others don't. For instance, supposing a thing's redness grounds its being coloured, we can wiggle the ground (by making the thing blue) without changing the grounded (the thing will still be coloured). According to this line of thought, it is misguided to intervene on the grounds, because the grounded doesn't counterfactually depend on the ground. The counterfactual dependence between ground and grounded runs in the opposite direction, so that if the grounded were to change (such that the thing were no longer coloured), the ground would thereby change as well (the thing would no longer be red). In such cases, the interventionist must wiggle the grounded and see whether the ground wiggles. ${ }^{12}$ To capture these sorts of cases, grounding interventionists might revise their view in the following way, which would make it structurally disanalogous with causal interventionism:

Grounding interventionism (disjunctive): $\quad \alpha$ grounds $\beta$ only if there is a possible intervention on $\alpha$ or $\beta$ that, were it carried out, would change the value of the other variable.

However, this revised approach arguably does not capture the motivation for the initial view, which was that grounds are like causes. It's causes we intervene on, since the arrow of determination flows from cause to effect, and that's the objective asymmetry we want to capture. The same goes, mutatis mutandis, for grounds. So grounding interventionists may insist that the view has to be formulated in

\footnotetext{
${ }^{11}$ For the formal details required to prevent this approach from being too permissive, see pp. $82-84$ in Woodward (2003).

12 I thank Fabrice Correia for bringing this to my attention.
} 
terms of possible interventions on grounds, while instead proposing special ways of bracketing or precluding preemption cases. ${ }^{13}$

Overdetermination and preemption aside, one might still think grounding interventionism is inherently confused. In particular, it may be argued that some cases of grounding appear to involve bi-directional metaphysical necessitation - and that there is a fundamental lack of fit between such cases and the interventionist paradigm. For instance, wiggling Socrates automatically wiggles Singleton Socrates and vice versa. Wiggling Singleton (whatever that might mean!) automatically wiggles Socrates. This suggests that some alleged grounding relata have a coarse-grained modal relationship that interventionist counterfactuals simply don't capture: the relata necessarily co-exist, so there is no asymmetric counterfactual dependence between them, and as such you cannot intervene on one independently of the other. One might suspect that this is a bug of the example at issue - but the Singleton example is generally taken to be paradigmatic of grounding!

The grounding formalism can allay some of these concerns. For instance, Schaffer explains: "the idea that one can 'surgically intervene' requires a kind of modularity condition on grounding, which corresponds to a free recombination assumption for the more fundamental", in which "one can adjust one of the more fundamental parameters while leaving the others as is" (2016, 71). We can represent Socrates and Singleton Socrates using distinct variables and encode their alleged asymmetric dependence into the equations we use to model them $(2016,73)$. This means that the formalism in which we enfold grounding "must tolerate" countermetaphysical and counterlogical scenarios $(2016,71)$, for which one needs a non-vacuous semantics. ${ }^{14}$ So we can force asymmetry into the grounding models. Whether this truly resolves the concern that some paradigm cases of grounding intuitively fail to exhibit asymmetric dependence is open to debate.

We have considered some initial formulations of grounding interventionism involving counterfactual interventions being necessary and sufficient for grounding, being sufficient only, targeting grounds only, and targeting either grounds or grounded facts/entities. I will not privilege any of the formulations that I have canvassed. My aim was just to consider some of the available options. In the process, I hope to have illuminated some initial choice-points, challenges, and potential bones of contention. I leave finer-grained articulations and defences of the view to its would-be proponents.

\section{Interventions}

Having sketched some basic formulations of grounding interventionism, I can now proceed to ask what, precisely, a grounding intervention could be. I will start by considering how causal

\footnotetext{
${ }^{13}$ For instance, on A. Wilson's (2018) view, with a suitably restrictive notion of intervention in which interventions cut off alternate grounding routes, the problem of preemption doesn't arise. In the case of the red object, the right kind of intervention makes the object colourless rather than blue. The right kind of intervention also precludes grounds from wiggling as a result of wiggling the grounded. If such an intervention were to make the thing colourless, the thing would (per impossibile) nevertheless remain red. I thank $\mathrm{Al}$ for raising these points in correspondence.

14 On the semantics of countermetaphysicals, see A. Wilson (2018a).
} 
interventionists have precisified their notion of an intervention. Again, I will take Woodward (2003) as the model. In his view, intervention is a token-level causal notion (2003, 98). Moreover, interventions need not be human manipulations; on the contrary, they bear no essential connection to human agency. Instead, an intervention on $X$ with respect to $Y$ changes the value of $X$ in such a way that if any change occurs to $Y$, "it occurs only in virtue of the change in the value of $X$ and not through some other causal route" $(2003,94)$. In other words, the intervention changes the value of $X$, such that any resulting change to $Y$ goes through $X$, as opposed to resulting from some cause that merely correlates with the intervention. Woodward also stipulates that the change to $X$ must be a result of the intervention alone $(2003,96)$. In essence, an intervention alone changes the value of $X$, which directly changes the value of $Y$.

It is well known that this conception of intervention invokes causation, for it is not clear what 'directly changing' the values of variables could be if not causal. As such, the notion of an intervention does not provide independent purchase on causation, and it makes causal interventionism circular. However, Woodward stresses that his intention is not to provide a reductive account; instead, he intends to non-trivially elucidate causal concepts, claims, and the relations among them (2009, 253-254). Moreover, he argues that the circularity in question is non-vicious (2009, 254-255).

In cashing out the notion of an intervention, Woodward also characterizes interventions in terms of ideal experiments. Interventions are what we would do ideally - i.e. if all practical impediments were removed - to test for causal relations $(2003,46)$. The appeal to ideal conditions is needed because interventions need not be practically possible. In fact, Woodward claims, they need not even be physically possible: "commitment to a manipulability theory leads unavoidably to the use of counterfactuals concerning what would happen under conditions that may involve violations of physical law" (emphasis removed, 2003, 132). For instance, we know that the gravitational attraction of the moon causes the behaviour of the tides. According to Woodward, while it may be physically impossible to change the position of the moon alone or to perform similar interventions, we can still assess what would happen in such counterfactual scenarios and be confident in the resultant causal and explanatory claims. The counterfactuals that capture causal relations will inevitably include such counternomics.

If the modal scope of the 'possible' in 'possible intervention' is not limited to practical possibility or physical possibility, where does that leave us? Woodward claims: "an intervention on $X$ with respect to $Y$ will be 'possible' as long as it is logically or conceptually possible for a process meeting the conditions for an intervention on $X$ with respect to $Y$ to occur" $(2003$, 132). This means that the relevant modal space is remarkably broad. The only counterfactuals that cannot provide a test of the truth of causal claims are "those for which we cannot coherently describe what it would be like for the relevant intervention to occur at all or for which there is no conceivable basis for assessing claims about what would happen under such interventions..." (2003, 132). As such, causal interventionism requires us to assess counterfactuals inhabiting some of the farthest reaches of modal space. This means that causal interventionism requires a powerful modal epistemology.

With a clearer sense of the notion of a causal intervention in hand, we can now consider what a grounding intervention might be. If causal and grounding interventions are analogous, then 
in the case of grounding, the relevant counterfactuals track token grounding relations. Let us say, closely following Woodward's characterization of causal interventions, that a grounding intervention on one variable with respect to another changes the value of the one in such a way that if any change occurs to the other, it occurs in virtue of that first change.

Note that, just as causal interventionists invoke causation in their conception of an intervention, grounding interventionists similarly invoke grounding. This is suggested by the presence of the 'in virtue of' locution in the characterization I just gave, which grounding theorists take to signal grounding (Fine 2012). If so, then just as causal interventionism does not give independent purchase on causation, grounding interventionism does not give independent purchase on grounding. It is open to grounding interventionists to respond, as Woodward does, by emphasizing the non-reductive nature of their view and by suggesting that its value is in its nontrivial elucidation of networks of concepts and claims pertaining to grounding.

Having just noted the presence of the 'in virtue of' locution in the characterization of a grounding intervention, we are well-positioned to see that grounding interventionism offers a unique response to a much-discussed puzzle in the metaphysics of grounding: the question of what, if anything, grounds the grounding facts. Understood as an ontological thesis, grounding interventionism suggests that each token grounding fact obtains in virtue of a corresponding possible intervention. That is to say, possible interventions ground the grounding facts. Here the grounding interventionist faces the additional question of what grounds those second-order grounding facts, and so on. This issue has been addressed in different ways. Following Bennett (2011), the interventionist might say that possible interventions ground the first-order, second-order, third-order grounding facts - and so on to infinity, resulting in a well-founded and non-vicious infinite regress. Alternatively, they might characterize the second-order (and higher) grounding facts as autonomous in Dasgupta's sense, i.e. "not apt for being grounded", such that "the question of what grounds them does not legitimately arise in the first place" (2014b, 563; see also 2016). I leave it to defenders of the view to determine how best to further address the puzzle.

The most pressing question now is what changing the value of the relevant variables amounts to. Let's start with the causal case. On one natural interpretation, to change the value of $X$ is just what it sounds like: to plug a particular value ( 0 or 1, let's say) in for $X$ in the relevant causal model. This is, in effect, to add a condition or supposition to the model. But this cannot be what Woodward has in mind, because that would make all of the interventions at issue physically and, indeed, practically possible - even easy! After all, I can set the values of the variables as I like. So the interventions at issue are not interventions on our models but on the world. Changing the variable $X$ must mean changing worldly circumstances in a manner at odds with the laws of physics. So let us say that, in the causal case, changing the value of $X$ amounts to making some event occur or not occur.

As for grounding, what changing the value of $\alpha$ ultimately amounts to will depend on what sorts of things can be grounds. This is a matter of disagreement. Schaffer (2009) takes grounding relata to be objects; others take them to be facts (Audi 2012, Dasgupta 2014a, and Fine 2012). If the relata are objects, then changing the value of $\alpha$ could mean bringing some object into or out of existence; in the case of facts, it could mean making some fact obtain or not obtain. 
To make matters more concrete, let me appeal to some familiar examples. Take the following grounding claims:

Disjunction: The truth of P grounds the truth of PvQ.

Singleton: The existence of Socrates grounds the existence of Singleton Socrates.

Euthyphro: God's desiring that P grounds its being good that P. (A. Wilson 2018b, 731-732)

In these examples, 'wiggling the ground' requires, respectively, making P false, removing Socrates from history, and making God desire differently. Just as we asked about whether and in what sense causal interventions are possible, we can ask the same of these grounding interventions. Assume for convenience the standard nested model of possibility space, in which physical possibility is nested within a space of metaphysical possibility, itself nested within a space of logical possibility. Regarding the disjunction case, the possibility of the intervention is indeterminate, since whether and in what sense it is possible to make P false depends on what P says. In the case of Socrates, it is physically impossible (I assume) for us, temporally situated as we are, to remove him from history. As such, the intervention in virtue of which the grounding relation holds inhabits the merely metaphysical space of possibility, outside the space of physical possibility.

As for God desiring differently, the details depend on the theology held fixed, but I take it no theist thinks anyone can make God do anything, given God's supposed omnipotence. It is an interesting question what sort of modality is at issue. For a human to force a change to God wouldn't break any physical laws, since (pace Spinoza) God is supposedly not part of the natural world they describe. One might think the intervention is metaphysically impossible or, for conceptual reasons, logically impossible. ${ }^{15}$ Perhaps, in an ideal experiment, the human intervener would be omnipotent, too. In that case, we get a variation of the paradox of omnipotence, which we might also interpret as a symptom of logical impossibility. Where grounding interventionism requires us to countenance counterlogicals, it takes us right off the standard map of modal space. These familiar examples suggest that grounding interventionism may require us to countenance counterpossible, countermetaphysical, and counterlogical interventions.

In fact, if one thinks that grounds are sometimes physically, metaphysically, or logically necessary - as grounding theorists surely do - then examples like this will proliferate, since wiggling such grounds requires doing the impossible, in the respective senses. Take the following claims:

1. The fact that nothing can travel faster than light grounds the fact that events occurring in different light cones are causally isolated.

2. The fact that water is $\mathrm{H}_{2} \mathrm{O}$ grounds the fact that this sample of $\mathrm{H}_{2} \mathrm{O}$ is a sample of water.

3. The law of non-contradiction grounds the fact that the statement 'whales are mammals' is either true or false but not both.

15 This example of an apparent physical possibility that is, at the same time, metaphysically impossible complicates matters by showing that we must refine either the standard characterization of physical possibility (in terms of consistency with physical law) or the standard nested picture of modal space. 
These are plausible candidates for grounding claims, because they involve a kind of non-causal explanation of particular facts by appeal to more general principles. Now, suppose we wiggle the grounds. Doing so would involve 1) making it possible for things to travel faster than light, 2) altering the essence of water, and 3) rendering false the law of non-contradiction. Arguably, the envisaged interventions are counternomic, countermetaphysical, and counterlogical interventions, respectively. ${ }^{16}$ If such examples are any indication, grounding interventionism requires us to countenance interventions that are possible only at the farthest reaches of modal space.

Let's take stock. Grounding interventions can be understood in a manner closely parallel to causal interventions, i.e. as changes to the value of variables representing grounding relata. Given that some of those interventions are impossible, 'changing the value of variables' has to be understood in worldly terms. Depending on one's conception of the grounding relata, this might mean adding or deleting something from the roster of existents, or making some fact obtain or fail to obtain. Just as causal interventionism requires that we assess counterfactuals whose antecedents are possible only in remote regions of modal space, grounding interventionism makes a similar demand. So both views ask us to make judgments about modally remote counterfactual scenarios. The next question I wish to address is: how might we do that?

\section{Knowledge of Interventionist Counterfactuals}

Since causal interventionism is the comparatively well-explored progenitor of grounding interventionism, it will be instructive to examine its epistemological credentials alongside those of grounding interventionism. So how does the causal interventionist think we achieve our epistemic aims — whether knowledge, justified belief, understanding, confirmation, learning or discovery — with regard to causation?

In the most epistemically ideal cases, we simply do the manipulations. That is the best way of finding out about causal dependencies. Where deriving a causal claim requires making a judgment about some practically possible intervention, sometimes it suffices to run inductions on prior

experience of type-identical or suitably similar causal relations (compare Roca-Royes 2017). Going beyond practical possibility, Woodward suggests that, as the sorts of creatures who can interact with and manipulate aspects of the world, we develop practices of causal inference and explanation, which we can extend from cases where manipulation is possible to cases where it's not $(2003,11)$. For physically possible interventions, our knowledge of the physical laws can guide our judgments.

\footnotetext{
${ }^{16}$ One inspired by Woodward (2003) might say that we have no clear conception of what these experimental interventions would involve, and so the corresponding counterfactuals lack a clear meaning or truth value. One might worry that this line of thought makes trouble for a significant proportion of grounding cases, in which case one might also worry that grounding interventionists have overextended the interventionist framework. In response, grounding interventionists could endorse a more permissive standard of meaningfulness for their counterfactuals, but doing so would not eliminate the problem of having to assess counterfactuals the antecedents of which one cannot clearly conceive. A better response would be to argue that we do have a clear conception of the required interventions and to elucidate it. I leave that task to them.
} 
However, these sorts of piecemeal methods will only get us so far, especially relative to the vast space of logically possible interventions to which the causal interventionist indexes causality.

Is there anything more general that we can say in the elucidation of an epistemology that can underwrite interventionist frameworks? Given the centrality of counterfactuals to interventionist frameworks, such an epistemology would be, largely, an epistemology of counterfactuals. So it will be illuminating to consider which sorts of epistemic resources might figure into the assessment of counterfactuals generally and interventionist counterfactuals in particular. I will consider three such resources: 1) imagination, 2) structural equation models, and 3) theoretical constraints.

\subsection{Imagination}

I begin with Williamson's (2007b) epistemology of counterfactuals, to which there have been numerous critical responses. ${ }^{17} \mathrm{My}$ aim here is not to address the independent feasibility of the account but to see how conjoining it with causal and grounding interventionisms affects their epistemic credentials. Williamson suggests that the story of counterfactual knowledge is developmental and evolutionarily un-mysterious. As individuals accrue experience over time, their experience conditions them into "patterns of expectation which are called on in [their] assessment of ordinary counterfactual conditionals" (2007b, 167). Importantly, counterfactual thinking often invokes the imagination, "radically informed and disciplined" by an empirical background of beliefs and an accompanying folk physics (2007b, 143). Against that background, imaginative simulation allows us to discern, for instance, that "[i]f the bush had not been there, the rock would have ended in the lake" $(2007 \mathrm{~b}, 142)$.

We can think of our process of imaginatively assessing the relevant counterfactuals as involving the performance, not of ordinary causal manipulations, but of imaginative interventions. In imaginative intervention, we imagine changing a variable (in the worldly sense discussed above) and imaginatively simulate what would result. I imaginatively remove the bush and simulate the resultant path of the rock. While 'imaginative intervention' may sound novel and fancy, ultimately, imaginative interventions are just thought experiments, in which we "work through the implications of scenarios" (Gendler 2010, 1-2). ${ }^{18}$ If so, we assess interventionist counterfactuals by running thought experiments.

Now, if imagination is the primary mode of epistemic access to counterfactuals, how good are the causal interventionist's and the grounding interventionist's chances of knowledge (or justification, or whatever) of causation and grounding, respectively? Williamson notes that our ability to handle mundane counterfactuals might not extend equally well to all reaches of modal space:

[W] may well be more reliable in evaluating counterfactuals whose antecedents involve small departures from the actual world than in evaluating those whose antecedents involve much larger departures. We may be correspondingly more reliable in evaluating the possibility of

\footnotetext{
17 See Casullo (2012), Gregory (2017), Jenkins (2008), Mallozzi (2021), Roca-Royes (2011), and Tahko (2012).

18 On the role of imagination in thought experiments, see also Arcangeli (2010) and Meynell $(2014,2018)$.
} 
everyday scenarios than of 'far-out' ones, and extra caution may be called for in the latter case. (2007b, 164)

The more familiar the imagined scenario, the more reliable imagination will be as a source of modal information; the less familiar, the less confidence we should have in its deliverances. ${ }^{19}$ Williamson therefore speaks approvingly of those philosophers who have comparatively low confidence in their "radically strange" imaginative exercises (2007b, 164). ${ }^{20}$

If Williamson is right, then his epistemology of counterfactuals makes the epistemic credentials of causal interventionism rather a mixed bag. That's because causal interventionists demand the assessment of both relatively mundane counterfactuals and relatively strange ones, and modal imagination is a better guide to the former than to the latter. ${ }^{21}$ The epistemic value of the imagination in a particular context depends largely on how the imagination is constrained in that context. $^{22}$ It is because the imagination is 'radically informed and disciplined' by experience that it is, on Williamson's view, plausibly reliable in the evaluation of mundane counterfactuals concerning things like rock trajectories - i.e. in situations where folk physics is adequate for our predictive purposes; it is because imagination lacks adequate constraint in less mundane cases that it is not obviously reliable in those cases. ${ }^{23}$ The upshot is that modal imagination has highly restricted adequacy conditions. When it comes to strange enough counternomics, ordinary experience doesn't adequately prepare the imagination, and assessing them requires us to stretch our imaginative capacities beyond their adequacy conditions.

What happens then? Recall the idea that our imaginative simulations of counterfactual scenarios are thought experiments. We can compare the epistemic constraints on those thought experiments with those on real-life scientific experiments:

In the case of actual experiments, the theory-relevant evidence generally takes the form of data concerning the behavior of the physical world under specific conditions; in the case of thought experiments, the theory-relevant evidence generally takes the form of intuitions (or predictions) concerning such behavior. (Gendler 2010, 2)

\footnotetext{
${ }^{19}$ Similarly, Nichols points out that it's less clear that we can trust the verdicts of imagination about absolute possibility and necessity, as compared with local risk and opportunity $(2006,253)$.

${ }^{20}$ Some conceive of metaphysics as an importantly imaginative endeavour. For instance, Godfrey-Smith (2012) characterizes metaphysics as model-building, which involves imaginative modification of reality. Paul (2012) likewise characterizes it as model-building, the methodology of which "should accommodate imagining", among other things (2012, $23 \mathrm{fn}$. 30). It is an interesting question whether such a picture demands an adjustment to our sense of the epistemic aims of metaphysics. McSweeney (forthcoming) considers just this sort of question when she argues that metaphysics is essentially imaginative and defends its value relative to the epistemic aim of understanding.

21 This makes sense evolutionarily, in that, as Nichols points out, "the connection between imagination and modal judgment presumably earned its keep by facilitating nomological modal judgments" $(2006,246)$.

22 On the notion of epistemic constraint, its role and significance, see Bryant (2021).

${ }^{23}$ However, there are exceptions. For instance, imagination sometimes does poorly in seemingly mundane cases, e.g. in cases where we attempt to imaginatively predict our own responses to situations (Maibom 2016).
} 
That is, in cases where empirical evidence cannot inform or constrain imaginative simulations, imagination frequently calls on antecedent intuitions. I will suppose that intuitions are 'gut feeling' judgments. Now, these moves are admittedly quick. Intuitions have been characterized in a number of ways. ${ }^{24}$ Moreover, the network of relations among intuitions, thought experiments, imagination, science and non-science is undoubtedly complex and deserves a deeper treatment than I can give here. ${ }^{25}$ On the definitional matter, I'm just stipulating. On the role of intuitions in thought experiments, I believe a natural characterization of what it is for a judgment to be made 'from the gut' is for it to be made without significant guidance from experience, among other forms of evidence and constraint (such as logical constraint, to be discussed further below). We lack such guidance in pervasive, weird metaphysical cases. Thus, we rely on intuition when thinking through such cases.

By invoking intuitions, causal interventionism invokes one of the primary epistemic resources of modal rationalism in a way that diminishes the appeal of the view. While such judgments are valuable in contexts of discovery, in contexts of justification, they are epistemically suspect for a number of familiar reasons. Some experimental evidence points to their cultural variation, and some suggests they are strongly vulnerable to cognitive bias. ${ }^{26}$ Moreover, science has a track record of repeatedly overturning common sense metaphysical intuitions (Shtulman and Harrington 2016), including even the strongest modal intuitions, such as the intuitions "that nonEuclidean geometry is impossible as a model of physical space, that it is impossible that there not be deterministic causation, [and] that non-absolute time is impossible" (Ladyman and Ross 2007, 16). Since surprise is a measure of scientific success (French and Murphy forthcoming), we might even think that counterintuitiveness is characteristic of scientific discovery. These points cast serious doubt on the evidential weight of intuitions. However, independently of these sorts of arguments, I believe that in many contexts - metaphysics included - there is little reason to take intuitions to be even defeasibly evidential in the first place. At the far reaches of modal space, where experience leaves imagination blind and counterfactual evaluation must invoke intuition, the causal interventionist's epistemology is rendered ineffectually rationalist.

As for grounding interventionism, it fares worse that its causal cousin when coupled with Williamson's epistemology of counterfactuals. In the causal case, imagination operates against a relatively rosy epistemological backdrop. We have (pace Hume) a great deal of empirically-based causal knowledge. As Woodward stresses (2003, 34), many causal dependencies are evidenced by

\footnotetext{
24 Booth and Rowbottom (2014), Cappelen (2012), Deutsch (2015), Devitt (2015), Dorr (2010), Gendler (2010), Nicoli (2016), Weinberg (2016).

25 See, for instance, Levy and Godfrey-Smith (2019).

26 On their cultural variability, see Beebe and Undercoffer (2016), Machery et al. (2004), Li et al. (2018), Nichols et al. (2003), and Weinberg et al. (2001); on their vulnerability to cognitive bias, see Andow (2016), Schwitzgebel and Cushman (2012), Swain et al. (2008), and Wheatley and Haidt (2005).
} 
actual experimentation. Prior experience of causal patterns reliably enables prediction. ${ }^{27}$ We also appear to have a hardwired capacity to identify instances of causation that "emerges early in development, and in some cases is remarkably fast and efficient" (Woodward 2003, 29). So the causal interventionist can capitalize on a great deal of prior working knowledge of causation.

By contrast, in the grounding case, the epistemological backdrop is less auspicious. The sorts of grounding relations typically at issue in metaphysics are distinctively metaphysical, composing a level of metaphysical structure that is not directly empirically discoverable, to which we have only indirect epistemic access. Neither prior experience nor empirical experimentation informs judgments about, for instance, abstract objects like Singleton Socrates or non-empirical matters like the goodness of an action. Neither should we hold our collective breath for studies in developmental psychology to show that we excel at identifying instances of grounding, since grounding is tied to practices of abstract metaphysical explanation rather than to the sphere of action and decisionmaking like its causal cousin. With grounding, we just don't have the epistemic traction we have with causation.

The fact is that the metaphysics of grounding gets murky and perplexing, fast. Some grounding interventions are 'radically strange', as Williamson put it. With countermetaphysicals, experience constrains the imagination even less than it does with counternomics - and leaves it even more reliant on intuitions that we have no good reason to believe are reliable or evidential. If so, then an imagination-based epistemology of counterfactuals renders grounding interventionism epistemically impoverished, and grounding interventionists must either defend the permissibility of stretching our imaginative resources beyond their adequacy conditions or point to some other mode of epistemic access to the relevant modal truths.

\subsection{Structural Equation Models}

Perhaps would-be grounding interventionists can benefit from an alternate (potentially complementary) tack, in which they co-opt formal tools that have been used fruitfully to model causal relations. In this vein, Schaffer claims that "the causal discovery algorithms associated with structural equation models furnish as precise and well-understood an epistemology as one could hope for" (2016, 67). Since those models are not inherently causal, Schaffer (2016) and A. Wilson (2018a, 2018b) are optimistic about the prospects of structural equation models to illuminate features of grounding structure and to reveal first-order grounding facts. Schaffer is careful to emphasize that the models are not a "magical panacea" but rather the best available formalism $(2016,61)$.

In essence, structural equation models comprise variables representing independent and dependent conditions (i.e. causes and effects; grounds and grounded entities or facts) and structural equations that say what the value of dependent variables will be given certain values for the independent ones. Modellers then say 'what actually happens' (Schaffer 2016, 62), by assigning

${ }^{27}$ Jansson (2018) likewise highlights how a posteriori knowledge of local causal matters constrains judgments of the aptness of causal models. 
values to the independent variables. The models then spit out values for the dependent variables, from which we read off causal or grounding claims.

Schaffer acknowledges various complicating factors here. First, as we have seen, it is controversial how best to read token relations off causal models — and by extension grounding models - in problem cases such as those involving preemption $(2016,65)$. In particular, some argue that structural equation models must distinguish between default and deviant variables, corresponding to expected and surprising events, respectively. ${ }^{28}$ In response, Blanchard and Schaffer (2017) argue that the distinction encodes a cognitive bias that influences causal judgment but that shouldn't be incorporated into models of causation itself.

Second, it is also a matter of debate how to handle the problem of underdetermination. As Schaffer explains, there can be multiple apt models of a situation, and they can disagree over causation. In such cases, it's not clear which causal claims we should commit to or why. While we might relativize causal claims to particular models, doing so eliminates the metaphysical objectivity we might have hoped for $(2016,68)$.

Finally, Schaffer acknowledges that like interventionism itself, structural equation models are also circular. That's because the division of variables into independent and dependent conditions and the parent-child asymmetry of the structural equations are built into the models $(2016,66)$. At any rate, it is clear that structural equation models don't make all substantive questions and difficulties disappear; on the contrary, they come with their own deep and difficult choice-points.

Let us suppose for argument's sake that, in time, these issues will be satisfactorily resolved. Other prospective problems still remain. For instance, Koslicki (2016) presents a number of reasons to doubt that structural equation models apply equally well to grounding as to causation. Among other things, she argues that prototypical cases of grounding like the determinate/determinable relation are problem cases for structural equation models because they are structurally similar to cases of massive causal preemption. For instance, Koslicki considers Schaffer's own example of the shirt's being maroon grounding the shirt's being red. She points out that the example is case in which wiggling the grounds need not wiggle the grounded, because supposing the shirt's color changes from maroon to crimson, "the shirt continues to be red, only in a different way" (2016, 107). So we have a case of grounding that is analogous to a case of massive causal preemption, in that the grounded can be brought about by multiple grounds, each of which can individually constitute a full ground and obtains only if the others don't obtain. Koslicki remarks:

At most, then, we are dealing with a situation in which a supposedly clear case of grounding is comparable to a problematic case of causation, one which has led to headaches for extant theories of causation including, by Schaffer's own admission, the structural equation model of causation. $(2016,108)$

If Koslicki is right that structural equation models render problematic erstwhile unproblematic cases of grounding, then we might doubt that structural equation models are really the key to a satisfactory grounding interventionist epistemology. In fact, from these and other concerns relating to the extension of structural equation models to grounding (e.g. those of Jansson 2018), one might

28 See for instance Menzies (2007), Hitchcock (2007), and Hall (2007). 
draw the conclusion that, far from being a beneficial component of a satisfactory epistemology of grounding, the interventionist framework makes theories of ground worse off epistemically than they would be otherwise. This is a stronger conclusion than I presently wish to defend, but I flag it as a live possibility.

At any rate, suppose again for the sake of argument that interventionists can adequately resolve this concern by developing a novel approach to preemption cases. ${ }^{29}$ If they were to do so, could we then say that structural equation models imbue grounding interventionism with adequate epistemological resources? I believe not.

To my mind, a central factor to consider in evaluating the potential of structural equation models to flesh out the grounding interventionist's epistemological story is that we don't just want to model causation and grounding; we want to model them well. It's clear that we need good reason to be confident of what we put in to our models - including, among other things, the functions that purportedly relate the variables - before we can justifiably be confident of what the models spit out. This much is uncontroversial. Indeed, Schaffer acknowledges the point explicitly:

I am not trying to show that merely by adopting the structural equations formalism, one gets the right answers to grounding questions for free. On the contrary: to get the right answers to grounding questions one has to put the right structural equations into the model, which encode the form and direction of dependency. $(2016,77)$

Likewise, one of Schaffer's aptness conditions for grounding models requires that the counterfactuals encoded in the model's equations be true $(2016,75)$. So the need for true (or justified, or known, or otherwise epistemically well-founded) starting counterfactuals is clear and acknowledged. But this immediately raises an important and more foundational epistemological question. Why should we think those counterfactuals are true (or justified, or known, etc.)? Grounding theorists may fill in this more foundational epistemological blank as they like. Yet the presence of such a blank shows that structural equation models can only supplement the grounding interventionist's epistemology. We need independent reasons for believing or accepting the counterfactuals we use to set up our structural equation models. So structural equation models cannot form the basis of an independently adequate epistemology of interventionist counterfactuals, nor are they intended to. Rather, they must complement some further and more foundational epistemic resource, in virtue of which we can be assured that we're setting up the relevant models well. The epistemic credentials of causal and grounding interventionism will then largely hang on the reliability of that resource.

\subsection{Theoretical Constraint}

One possibility is that certain theoretical constraints could provide the needed epistemic resource. I have discussed the nature and importance of such constraints elsewhere (Bryant 2021), but the general idea is simple and familiar: holding fixed certain background claims restricts the sorts of further claims we can make. In the interventionist context, one might hope that background

\footnotetext{
${ }^{29}$ For instance, by taking A. Wilson's (2018) approach to such cases (see footnote 10).
} 
theory can appropriately constrain counterfactual assessment. Three main candidates immediately suggest themselves: background science, background logic, and background metaphysics.

First, let's consider whether science can adequately constrain the assessment of interventionist counterfactuals. Here, we might take inspiration from certain causal interventionists. For instance, in their manipulationist account of causation, Menzies and Price appeal to artificial simulations to make sense of how we can justify causal claims about unmanipulable events. Take the claim that some earthquake was caused by friction between continental plates. Menzies and Price argue:

We can make such causal claims because we believe that there is another situation that models the circumstances surrounding the earthquake in the essential respects and does support a means-end relation between an appropriate pair of events. The paradigm example of such a situation would be that created by seismologists in their artificial simulations of the movement of continental plates. $(1993,197)$

The simulation informs judgments about the relevant counterfactuals because it adequately models the mechanics of earthquakes. When artificial simulations are founded on a rich body of data and have a track record of predictive success, they can significantly aid counterfactual reasoning.

That's fine for the physical possibilities, but matters get trickier when it comes to counternomics. Woodward is cognizant of the epistemological worries that arise from his move toward conceptual or logical possibility. He admits:

It is arguable that as we make the relevant notion of 'possible intervention' more and more permissive, so that it includes contra-nomic possibilities and so on, we reach a point at which this notion and the counterfactuals in which it figures become so unclear that we can no longer use them to illuminate... causal claims. $(2009,256)$

That's because it is unclear what the proposed intervention would involve and how to determine what would result from it $(2009,257)$. If so, then perhaps counternomics might be thought to take us beyond the "natural range of application" of causal interventionism (2009, 256).

However, Woodward suggests that, at least in some cases, elements of well-confirmed scientific theories constrain our judgment with regard to physical impossibilities. Recall the example of the moon's gravitational attraction and its effect on the tides. Woodward suggests:

Although it may be true that any actual physical process that changes the position of the moon will also directly influence the tides, Newtonian theory and familiar rules about the composition of forces tell us how to subtract out any direct influence from such a process so that we can calculate just what the effect of, say, doubling of the moon's orbit (and no other changes) would be on the tides, even though it also may be true that there is no way of actually realizing this effect alone. $(2003,131)$

That is, the appropriate physical theory can help us to establish the right kind of modularity in our model of the intervention and its consequences. Woodward continues:

Newtonian theory itself delivers a determinate answer to questions about what would happen to the tides under an intervention that doubles the moon's orbit, and this is enough for counterfactual claims about what would happen under such interventions to be legitimate and to allow us to assess their truth. $(2003,131)$ 
So science can constrain our assessments of at least some counternomics.

Does this approach give causal interventionism adequate epistemic footing? The example works because it involves isolating variables that wouldn't normally be isolable in an otherwise familiar possible world; it's less clear that science can guide inferences about worlds where the physical laws differ more significantly from those of the actual world. At any rate, when causal interventionism invokes (as it inevitably does) counterfactuals with merely logically or conceptually possible antecedents, science doesn't obviously help. So the appeal to scientific models and background theory as a means of answering questions about counternomic scenarios may go some way to allaying concerns about the epistemic credentials of causal interventionism, but it won't provide a completely satisfying story.

As for grounding interventionism, scientific models and background theory are likewise powerless to constrain our reasoning about certain paradigmatic metaphysical cases. ${ }^{30}$ For instance, science has no clear bearing on what would happen to Singleton Socrates had Socrates not existed or to the good had God desired differently. As such, while the framework of interventionism does possess a moderate glow of scientific respectability (owing in part to its intimate ties with causal modelling), it does not impart that same glow to standard-fare metaphysics of ground. That is to say, at the risk of bursting certain bubbles, if causal interventionism has reasonably good naturalistic credentials, theories of ground don't necessarily improve their own naturalistic standing just by coopting interventionist language and resources.

While science doesn't appear to bear on the standard metaphysical cases, it might be thought that a special class of semi-empirical grounding claims can be drawn from science and usefully modelled within the interventionist framework. While it may not be obvious at first glance, science does have some relevance to questions of ground (Bryant 2018), which is evidenced by other sorts of example. For instance, suppose that being an electron grounds having negative charge (Audi 2012, 117). Suppose also that $x$ is an electron. To test this grounding claim, the grounding interventionist would have us assess the following counterfactual: if an intervention were to remove the property of being an electron from $x, x$ would no longer have negative charge. Now, science does bear on the assessment of this counterfactual, by furnishing us with requisite background information about the characteristic properties of electrons. Science also tells us that other sorts of particles can be negatively charged. However, metaphysics rears its head when we ask whether $x$ might have been one of those other sorts of particles. So scientific background theory may help to constrain our assessments of some such grounding counterfactuals, but murky metaphysical questions quickly intrude.

We may also wonder what proportion of grounding counterfactuals will be amenable to scientific constraint. As I noted above, grounding theorists commonly posit grounding relations among abstract, non-empirical relata, where scientific models and background theory are not clearly

\footnotetext{
30 Jansson (2018) argues, similarly, that while our assessments of the aptness of causal models are constrained by local, a posteriori theories of causal mechanisms and processes, there is no analogous a posteriori constraint on our judgments of the aptness of grounding models. While I agree with the general sentiment, I don't think the point holds universally; as the next paragraph will make clear, empirical and scientific background theory provides at least partial epistemic constraint in some cases of grounding.
} 
relevant. At any rate, as we have seen, the epistemology gets a lot murkier when we come to far out counterfactuals such as countermetaphysicals. As I noted in the case of causal interventionism, when we assess certain modally remote counterfactuals, the door is open for scientifically unmoored intuitions to sneak into modal judgment. The greater the evidential role those intuitions play, the more suspect the epistemology of grounding interventionism will be.

To the extent that grounding interventionists wish to reason about counterfactuals to which scientific background theory offers little guidance, those interventionists would do well to invoke further constraints. For instance, one might wonder whether logic can constrain the assessment of grounding counterfactuals. Indeed it can — and this is relatively straightforward in certain cases. For instance, so long as $\mathrm{P}$ and $\mathrm{Q}$ aren't necessarily true statements, wiggle $\mathrm{P}$ and you wiggle $\mathrm{P} \wedge \mathrm{Q}$. Even in more modally remote cases, where we must consider counterlogicals, our reasoning can be well constrained by clear formal frameworks for reasoning in the face of contradictions. This shows that it's not modal remoteness per se that is most epistemically relevant factor, but how well our epistemic resources constrain counterfactual assessment. At any rate, the results of interventions that follow as a straightforward matter of logic are one thing; the results of interventions in meatier metaphysical matters are another. Does wiggling the part wiggle the whole, the brain state the mental state, the determinate the determinable, the truthmaker the truth, the natural properties the moral properties...? Our theories concerning such matters are subject to logical constraints just like any other theories, but logic radically underdetermines the answers to these questions and thus does not constitute an adequately robust constraint of its own accord (see Bryant 2020). I suggest that the straightforward cases in which logic adequately constrains the assessment of grounding counterfactuals don't constitute a particularly large swath of the sorts of counterfactuals that theorists of ground are interested in.

Perhaps a more substantive form of constraint can come from background metaphysical theory. Once again, there are relatively straightforward cases of this. If we hold fixed a metaphysics according to which sets supervene on their members, that metaphysics has clear implications for what happens when we wiggle Socrates. If we hold fixed a metaphysics according to which God's will uniquely determines the good, that metaphysics has clear implications for what happens when we wiggle God's will. Note that the imagination isn't invoked in these cases; all that's needed is an understanding of the relevant metaphysical principles and relations, as well as some basic logical capacities.

However, we need to know a fair bit about the relevant dependence relations before we can perform the relevant counterfactual assessments - we need to know that sets supervene on such-andsuch and that God's will uniquely determines so-and-so. ${ }^{31}$ So it's not clear - at least from these initial paradigm cases - to what extent this approach fosters the discovery of new information about hierarchical structure. What is clear is that appeal to metaphysical constraint makes grounding interventionism an exercise in determining what follows from our antecedent metaphysical commitments, not a direct interrogation of objective grounding structure. Some might think that's a perfectly acceptable place to situate theories of ground in the epistemic scene and a perfectly fine

31 If grounding critics like J. Wilson (2014) and Koslicki (2015) are right, in having such knowledge, we have a finer-grained understanding of the dependence at issue than we do by positing a grounding relation. 
characterization of what grounding theorists are up to. But for those grounding theorists with loftier epistemic aims, this may be an unacceptable limitation of the approach.

It's also worth emphasizing that what gets held fixed as the metaphysical background theory is relatively open - perhaps too open for us to be assured that we're engaging effectively in a truthseeking activity. While there is relative consensus about many matters in science (which is, of course, complemented by much substantive disagreement, e.g. about matters of theoretical interpretation and unification), there is comparatively far less consensus on matters metaphysical (which isn't to say there is no such consensus). Most things are up for grabs in metaphysics, including even foundational assumptions and basic conceptual matters. So in many cases, how one fixes the metaphysical background will depend on one's metaphysical proclivities and allegiances. This makes grounding interventionism no more factional than the rest of metaphysics, but it does somewhat quash any latent hope that the interventionist framework might lend increased objectivity to theories of ground.

\section{Conclusion}

The aim of this paper was to consider the prospects for an adequately formulated and epistemologically well-founded grounding analog of causal interventionism. I considered several formulations of grounding interventionism, in which grounding relations are (non-reductively) cashed out in terms of possible interventions on variables representing grounding relata. A grounding intervention on one variable with respect to another changes the value of the one in such a way that if any change occurs to the other, it occurs in virtue of the change in the value of the one. We saw that such interventions should be understood in worldly terms, as adding something to or deleting something from the roster of entities, or making some fact obtain or fail to obtain.

After considering candidate formulations of the view, I turned to the evaluation of its epistemic credentials. This involved considering how well imagination, structural equation models, and background theory constrain our assessments of interventionist counterfactuals. I argued that causal interventionism and grounding interventionism both demand that we stretch our imaginative capacities beyond their adequacy conditions when they ask us to assess certain modally remote counterfactuals that neither experience nor other forms of evidence and constraint adequately prepare us to assess. In such cases, imagination relies on unchecked intuition. Moreover, I argued that structural equation models merely formalize causal and grounding assumptions that require independent justification. Finally, I argued that while certain forms of background theory can help constrain the assessment of some causal and grounding counterfactuals, our epistemic grip weakens relatively quickly. In the trickier cases, counterfactual reasoning again invokes unfettered intuitions. Just how satisfied we should be with these epistemological options depends on the proportion of causal and grounding counterfactuals whose assessment is robustly constrained relative to those whose assessment isn't. In my view, the available epistemological options for causal interventionism and grounding interventionism are insufficiently powerful relative to the full spectrum of cases philosophers tend to be are interested in - especially so for cases of grounding. As such, grounding 
interventionism requires firmer epistemological foundations if it is to be a viable and attractive theoretical alternative.

\section{Acknowledgments}

This research was funded by Portuguese national funds through Fundação para a Ciência e a Tecnologia, I.P., in the scope of the project PTDC/FER-HFC/30665/2017. I thank Al Wilson, Nick Emmerson, Noelia Iranzo Ribera, the eidos research group, and two anonymous referees for remarkably helpful feedback on a draft of the paper. For helpful discussion, I also thank the audience at my presentation of this work at New Directions in Metaphilosophy at the University of Kent.

\section{References}

Andow, J. (2016) 'Reliable But Not Home Free? What Framing Effects Mean for Moral Intuitions', Philosophical Psychology, 6, pp. 1-8.

Arcangeli, M. (2010) 'Imagination in Thought Experimentation: Sketching a Cognitive Approach to Thought Experiments', in Magnani, L., Carnielli, W., and Pizzi, C. (eds) Model-Based Reasoning in Science and Technology. Dordrecht: Springer, pp. 571-587.

Audi, P. (2012) 'A Clarification and Defense of the Notion of Grounding', in Correia, F. and Schnieder, B. (eds) Metaphysical Grounding: Understanding the Structure of Reality. Cambridge: Cambridge UP, pp. 101-121.

Beebe, J. R. and Undercoffer, R. (2016) 'Individual and Cross-Cultural Differences in Semantic Intuitions: New Experimental Findings', Journal of Cognition and Culture, 16(3-4), pp. 322357.

Bennett, K. (2011) 'By Our Bootstraps’, Philosophical Perspectives, 25(1), pp. 27-41.

Blanchard, T. and Schaffer, J. (2017) 'Cause without Default', in Beebee, H., Hitchcock, C., and Price, H. (eds) Making a Difference. Oxford: Oxford University Press, pp. 175-214.

Booth, A. R. and Rowbottom, D. P. (eds) (2014) Intuitions. Oxford: Oxford University Press.

Bryant, A. (2021) 'Epistemic Infrastructure for a Scientific Metaphysics', Grazer Philosophische Studien, 98, pp. 27-49.

Bryant, A. (2020) 'Keep the Chickens Cooped: The Epistemic Inadequacy of Free Range Metaphysics', Synthese, 197, pp. 1867-1887.

Bryant, A. (2018) 'Naturalizing Grounding: How Theories of Ground Can Engage Science', Philosophy Compass, 13:e12489.

Byrne, R. (2007) The Rational Imagination. Cambridge, MA: MIT Press.

Cappelen, H. (2012) Philosophy without Intuitions. Oxford: Oxford University Press.

Casullo, A. (2012) 'Counterfactuals and Modal Knowledge', in Essays on a Priori Knowledge and Justification. New York: Oxford University Press, pp. 251-270. 
Correia, F. (2014) 'Logical Grounds', The Review of Symbolic Logic, 7(1), pp. 31-59.

Dasgupta, S. (2017) 'Constitutive Explanation’, Philosophical Issues, 17(1), pp. 74-97.

Dasgupta, S. (2016) 'Metaphysical Rationalism’, Nous, 50(2), pp. 379-418.

Dasgupta, S. (2014a) 'On the Plurality of Grounds’, Philosophers' Imprint, 14, pp. 1-28.

Dasgupta, S. (2014b) 'The Possibility of Physicalism', Journal of Philosophy, 111(9-10), pp. 557-92.

DeRosset, L. (2014) 'Getting Priority Straight', Philosophical Studies, 149(1), pp. 73-97.

Deutsch, M. (2015) The Myth of the Intuitive: Experimental Philosophy and Philosophical Method. Cambridge, MA: MIT Press.

Devitt, M. (2015) 'Relying on Intuitions: Where Cappelen and Deutsch Go Wrong', Inquiry, 58(7-8), pp. 669-699.

Dorr, C. (2010) 'Review of Every Thing Must Go: Metaphysics Naturalized', Notre Dame Philosophical Review. Available at: https://ndpr.nd.edu/news/24377-every-thing-must-go-metaphysicsnaturalized/.

Fine, K. (2012) 'Guide to Ground', in Correia, F. and Schnieder, B. (eds) Metaphysical Grounding. Cambridge: Cambridge UP, pp. 37-80.

French, S. and Murphy, A. (forthcoming) 'The Value of Surprise in Science', Erkenntnis.

Frisch, M. (2014) Causal Reasoning in Physics. Cambridge: Cambridge University Press.

Gendler, T. (2010) Intuition, Imagination, and Philosophical Methodology. Oxford: Oxford University Press.

Glazier, M. (2020) 'Explanation', in Raven, M. (ed.) Routledge Handbook of Metaphysical Grounding. New York: Routledge, pp. 121-132.

Godfrey-Smith, P. (2012) 'Metaphysics and the Philosophical Imagination', Philosophical Studies, 160, pp. 97-113.

Gregory, D. (2017) 'Counterfactual Reasoning and Knowledge of Possibilities', Philosophical Studies, 174(4), pp. 821-835.

Hall, N. (2007) 'Structural Equations and Causation’, Philosophical Studies, 132, pp. 109-136.

Hitchcock, C. (2007) 'Prevention, Preemption, and the Principle of Sufficient Reason', Philosophical Review, 116(495-532).

Hitchcock, C. and Woodward, J. (2003) 'Explanatory Generalizations, Part II: Plumbing Explanatory Depth', Nous, 37(2), pp. 181-199.

Hoerl, C., McCormack, T. and Beck, S. (eds) (2011) Understanding Counterfactuals, Understanding Causation: Issues in Philosophy and Psychology. Oxford: Oxford University Press.

Ichikawa, J. (2011) 'Quantifiers, Knowledge, and Counterfactuals', Philosophy and Phenomenological Research, 82(2), pp. 287-313. 
Jansson, L. (2018) 'When are Structural Equation Models Apt? Causation Versus Grounding', in Reutlinger, A. and Saatsi, J. (eds) Explanation Beyond Causation: Philosophical Perspectives on NonCausal Explanations. Oxford: Oxford University Press, pp. 250-266.

Jenkins, C. (2008) 'Modal Knowledge, Counterfactual Knowledge and the Role of Experience', Philosophical Quarterly, 58(223), pp. 693-701.

Koslicki, K. (2016) 'Where Grounding and Causation Part Ways: Comments on Schaffer', Philosophical Studies, 173, pp. 101-112.

Koslicki, K. (2015) 'The Coarse-Grainedness of Grounding', in Bennett, K. and Zimmerman, D. (eds) Oxford Studies in Metaphysics, Volume 9. Oxford: Oxford UP, pp. 306-44.

Kroedel, T. (2017) 'Modal Knowledge, Evolution, and Counterfactuals', in Fischer, R. and Leon, F. (eds) Modal Epistemology After Rationalism. Dordrecht: Springer International Publishing, pp. 179-195.

Kroedel, T. (2012) 'Counterfactuals and the Epistemology of Modality', Philosophers' Imprint, 12(12), pp. 1-14.

Ladyman, J. and Ross, D., with Spurrett, D. And Collier, J. (2007) Every Thing Must Go: Metaphysics Naturalized. Oxford: Oxford UP.

Leuenberger, S. (2014) 'Grounding and Necessity', Inquiry: An Interdisciplinary Journal of Philosophy, 57(2), pp. 151-174.

Levy, A. and Godfrey-Smith, P. (eds) (2019) The Scientific Imagination. Oxford: Oxford University Press.

Li, J. Longgen, L., Chalmers, E., and Snedeker, J. (2018) 'What is in a name?: The development of cross-cultural differences in referential intuitions', Cognition, 171, pp. 108-111.

Machery, E. Mallon, R., Nichols, S., and Stich, S. (2004) 'Semantics, Cross-Cultural Style', Cognition, 92(3), pp. B1-B12.

Mallozzi, A. (2021) 'Superexplanations for Counterfactual Knowledge', Philosophical Studies, 178(4), pp. 1315-1337.

Mandel, D., Hilton, D. and Catellani, P. (eds) (2005) The Psychology of Counterfactual Thinking. London: Routledge.

Maurin, A.-S. (2019) 'Grounding and Metaphysical Explanation: It's Complicated', Philosophical Studies, 176(6), pp. 1573-1594.

McSweeney, M. (forthcoming) 'Metaphysics as Essentially Imaginative and Aiming at Understanding', American Philosophical Quarterly.

Meek, C. and Glymour, C. (1994) 'Conditioning and Intervening', British Journal for the Philosophy of Science, 45(4), pp. 1001-1021.

Menzies, P. (2007) 'Causation in Context', in Price, H. and Corry, R. (eds) Causation, Physics, and the Constitution of Reality: Russell's Republic Revisited. Oxford: Oxford University Press, pp. 191223. 
Menzies, P. and Price, H. (1993) 'Causation as a Secondary Quality', British Journal for the Philosophy of Science, 44(2), pp. 187-203.

Meynell, L. (2018) 'Images and Imagination in Thought Experiments', in Stuart, M., Fehige, Y., and Brown, J. R. (eds) The Routledge Companion to Thought Experiments. London: Routledge, pp. 498-511.

Meynell, L. (2014) 'Imagination and Insight: A New Account of the Content of Thought Experiments', Synthese, 191(17), pp. 4149-4168.

Nichols, S. (2006) The Architecture of the Imagination: New Essays on Pretence, Possibility, and Fiction. Oxford: Oxford University Press.

Nichols, S., Stich, S. and Weinberg, J. (2003) 'Metaskepticism: Meditations in Ethnoepistemology', in Luper, S. (ed.) The Skeptics. Burlington: Ashgate Publishing, pp. 227-247.

Nicoli, S. M. (2016) The Role of Intuitions in Philosophical Methodology. London: Palgrave Macmillan.

Paul, L. A. (2012) 'Metaphysics as Modeling: The Handmaiden's Tale', Philosophical Studies, 160, pp. 129.

Paul, L. A. (2009) 'Counterfactual Theories', in Beebee, H., Hitchcock, C., and Menzies, P. (eds) The Oxford Handbook of Causation. Oxford: Oxford University Press, pp. 158-184.

Pearl, J. (2000) Causality: Models, Reasoning, and Inference. New York: Cambridge University Press.

Raven, M. (2015) 'Ground', Philosophy Compass, 10(5), pp. 322-333.

Roca-Royes, S. (2017) 'Similarity and Possibility: An Epistemology of De Re Possibility for Concrete Entities', in Leon, F. and Fischer, R. (eds) Modal Epistemology After Rationalism. Cham: Springer, pp. 221-245.

Roca-Royes, S. (2011) 'Modal Knowledge and Counterfactual Knowledge', Logique et Analyse, 54(216), pp. 537-552.

Rosen, G. (2010) 'Metaphysical Dependence: Grounding and Reduction', in Hale, R. and Hoffman, A. (eds) Modality: Metaphysics, Logic, and Epistemology. New York: Oxford UP, pp. 109-136.

Schaffer, J. (2017) 'Laws for Metaphysical Explanation', Philosophical Issues, 27, pp. 302-321.

Schaffer, J. (2016) 'Grounding in the Image of Causation', Philosophical Studies, 173, pp. 49-100.

Schaffer, J. (2009) 'On What Grounds What', in Manley, D., Chalmers, D., and Wasserman, R. (eds) Metametaphysics: New Essays on the Foundations of Ontology. Oxford: Oxford UP, pp. 347-383.

Schulz, M. (2017) Counterfactuals and Probability. Oxford: Oxford University Press.

Schwitzgebel, E. and Cushman, F. (2012) 'Expertise in Moral Reasoning? Order Effects on Moral Judgment in Professional Philosophers and Non-Philosophers', Mind and Language, 27(2), pp. 135-153.

Shtulman, A. and Harrington, K. (2016) 'Tensions Between Science and Intuition Across the Lifespan', Topics in Cognitive Science, 8, pp. 118-137. 
Skiles, A. (2015) 'Against Grounding Necessitarianism’, Erkenntnis, 80(4), pp. 717-751.

Spirtes, P., Glymour, C. and Scheines, R. (2000) Causation, Prediction and Search. Cambridge, MA: MIT Press.

Swain, S., Alexander, J. and Weinberg, J. (2008) 'The Instability of Philosophical Intuitions: Running Hot and Cold on Truetemp', Philosophy and Phenomenological Research, 76(1), pp. 138-155.

Tahko, T. (2012) 'Counterfactuals and Modal Epistemology', Grazer Philosophische Studien, 86(1), pp. 93-115.

Thompson, N. (2016) 'Grounding and Metaphysical Explanation', Proceedings of the Aristotelian Society, 116(3), pp. 395-402.

Trogdon, K. (2013) 'Grounding: Necessary or Contingent?', Pacific Philosophical Quarterly, 94(4), pp. 465-485.

Wang, J. (2020) 'Cause', in Raven, M. (ed.) The Routledge Handbook of Metaphysical Grounding. New York: Routledge, pp. 300-311.

Weinberg, J. (2016) 'Intuitions', in The Oxford Handbook of Philosophical Methodology. Oxford: Oxford University Press, pp. 287-308.

Weinberg, J., Nichols, S. and Stich, S. (2001) 'Normativity and Epistemic Intuitions', Philosophical Topics, 29(1-2), pp. 429-460.

Wheatley, T. and Haidt, J. (2005) 'Hypnotic Disgust Makes Moral Judgments More Severe', Psychological Science, 16(10), pp. 780-784.

Williamson, T. (2007a) 'Philosophical Knowledge and Knowledge of Counterfactuals', Grazer Philosophische Studien, 74(1), pp. 89-123.

Williamson, T. (2007b) The Philosopby of Philosophy. Oxford: Blackwell Pub.Williamson, T. (2020) 'Knowing by Imagining', in Boghossian, P. and Williamson, T. (eds) Debating the A Priori. Oxford: Oxford University Press, pp. 175-185.

Wilson, A. (forthcoming) 'Counterpossible Reasoning in Physics (Preprint)', Philosophy of Science, 88(5), pp. 1-20.

Wilson, A. (2018a) 'Grounding Entails Counterpossible Non-Triviality', Philosophy and Phenomenological Research, 96(3), pp. 716-728.

Wilson, A. (2018b) 'Metaphysical Causation', Nous, 52, pp. 723-51.

Wilson, J. (in progress) On the Notion of Diachronic Emergence.

Wilson, J. (2014) 'No Work for a Theory of Grounding’, Inquiry, 57(5-6), pp. 535-579.

Woodward, J. (2009) 'Agency and Interventionist Theories', in Beebee, H., Hitchcock, C., and Menzies, P. (eds) The Oxford Handbook of Causation. Oxford: Oxford University Press, pp. 234-263.

Woodward, J. (2003) Making Things Happen: A Theory of Causal Explanation. Oxford: Oxford UP. 
Woodward, J. (2000) 'Explanation and Invariance in the Special Sciences', The British Journal for the Philosophy of Science, 51(2), pp. 197-254.

Woodward, J. (1997) 'Explanation, Invariance, and Intervention', Philosophy of Science, 64, pp. S26S41.

Woodward, J. and Hitchcock, C. (2003) 'Explanatory Generalizations, Part I: A Counterfactual Account', Nous, 37(1), pp. 1-24. 